\title{
New experimental constraints on the electron screening effect in astrophysical plasma
}

\author{
N. Targosz-Sleczka*† \\ Institute of Physics, University of Szczecin, Szczecin, Poland \\ E-mail: natalia.targosz@wmf.univ.szczecin.pl \\ K. Czerski \\ Institute of Physics, University of Szczecin, Szczecin, Poland \\ Institute for Solid-State Nuclear Physics, Berlin, Germany \\ Institut für Optik und Atomare Physik, Technische Universität Berlin, Berlin, Germany
}

\section{A. Huke}

Institute for Solid-State Nuclear Physics, Berlin, Germany

Institut für Optik und Atomare Physik, Technische Universität Berlin, Berlin, Germany

\section{Kaczmarski, A. i. Kilic, D. Weissbach}

Institute of Physics, University of Szczecin, Szczecin, Poland

\section{G. Ruprecht}

Institute for Solid-State Nuclear Physics, Berlin, Germany

Study of the $d+d$ reactions at very low energies in metallic environments in the terrestrial laboratories, enables us to determine the strength of the screening effect in the strongly coupled astrophysical plasma in stars. So far, experimentally determined screening energies were extremely high in comparison to the theoretical predictions, and the reason for the observed discrepancies remained unrecognized. New measurements of the electron screening effect in ${ }^{2} \mathrm{H}(d, p)^{3} \mathrm{H}$ and ${ }^{2} \mathrm{H}(d, n){ }^{3} \mathrm{H}$ reactions at energies from 6 to $25 \mathrm{keV}$ were performed in zirconium under ultra-high vacuum conditions. The target surface contamination and the deuteron density have been continuously monitored. Precisely determined energy dependence of the screening enhancement factor deviates significantly from the single-parametric curve fitted only with the screening energy. To explain the experimental data it was necessary to include an additional contribution resulting from a hypothetical $0^{+}$threshold resonance in ${ }^{4} \mathrm{He}$ of a single-particle structure. The resonance component could be calculated as a coherent superposition with transition matrix elements for the dd reactions, reducing the value of experimentally obtained screening energy. However, its absolute value still overestimates the theoretical limit and can additionally be influenced by a number of crystal lattice defects of the metallic target.

XIII Nuclei in the Cosmos,

7-11 July, 2014

Debrecen, Hungary

\footnotetext{
* Speaker.

${ }^{\dagger}$ This work was partially supported by the Polish NCN grant no 2011/03/N/ST2/03627.
} 


\section{Introduction}

Low-energy nuclear reactions taking place in dense astrophysical plasmas are enhanced due to electron screening of the Coulomb barrier. The conceptual model for plasma used in laboratory investigation is $d+d$ reaction in metal. The experiments driven first under high vacuum (HV) conditions, showed that the experimentally determined screening energies corresponding to the reduction of the Coulomb barrier were by at least a factor of two larger than the theoretical values calculated in terms of the self-consistent dielectric function theory [1-3]. The enhanced screening effect in metals has been experimentally confirmed by many groups [4-6]. However, the comparison between experimental and theoretical data is difficult because of some systematic errors in experiments, resulting especially from oxidation of the target surface during the measurements. A series of new experiments dealing with atomically clean targets under ultra-high vacuum (UHV) conditions $[7,8]$ result in screening energies larger than the previous experimental values, escalating the discrepancy to the theoretical expectations. Thus, we discuss here an alternative source of the experimentally measured enhanced screening effect resulting from a narrow resonance close to the reaction threshold.

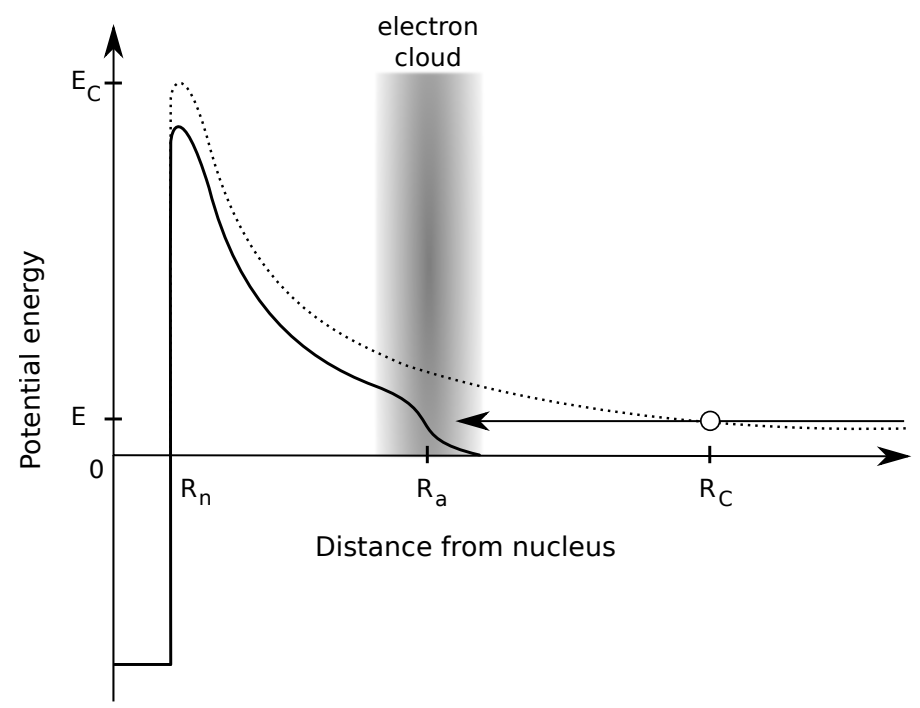

Figure 1: The penetration factor through the screened Coulomb barrier can be calculated by adding the screening energy $U_{e}$ to the projectile energy.

\section{Research}

The strength of the electron screening effect is described by the screening energy $U_{e}$, corresponding to the reduction of the Coulomb barrier. The potential is screened due to the fact that in metallic environment an electron cloud surrounds the reacting ions (Figure 1). For the $d+d$ reactions in metals the cross section $\sigma$ is measured. Our differential data analysis extracts all the details from the raw data by defining the screened reaction cross section [3]: 


$$
\sigma_{\mathrm{scr}}=\sigma_{\mathrm{bare}} \frac{P\left(E+U_{e}\right)}{P(E)}
$$

where

$$
P(E)=\sqrt{\frac{E_{G}}{E}} \exp \left(-\sqrt{\frac{E_{G}}{E}}\right)
$$

denotes the penetration factor, with the Gamow energy $E_{G}=986 \mathrm{keV}$ for dd reactions. With the cross sections from a precise gas target measurement [9] and low energy dependence of the stopping power $\frac{d E}{d x} \propto \sqrt{E}[10]$ one can present a possible screening enhancement by the thick target enhancement factor [3]:

$$
F(E)=\frac{\int_{0}^{E} \frac{\sigma_{\mathrm{scr}}(E)}{\sqrt{E}}}{\int_{0}^{E} \frac{\sigma_{\mathrm{bare}}(E)}{\sqrt{E}}} .
$$

Experimental screening energies, obtained previously under high vacuum conditions, in comparison with the theoretically predicted are presented in Figure 2 for different metals. The difference between the theoretical and experimental data is clearly visible. New measurements, obtained under UHV conditions yielded $U_{e}=497 \pm 3 \mathrm{eV}$, thus increasing the difference between theoretical and experimental data [8].

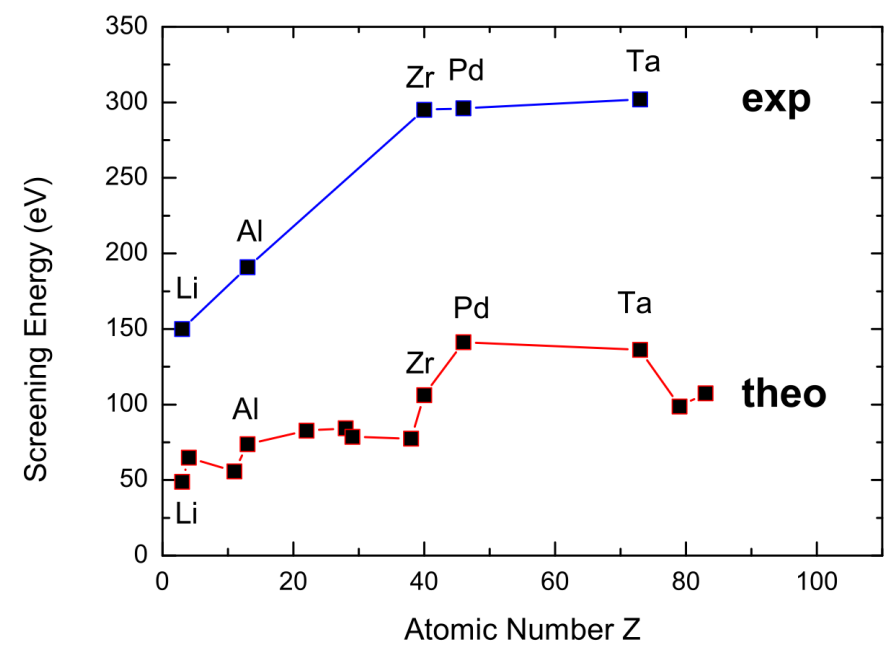

Figure 2: The HV experimental screening energies for different metals compared to the theoretical predictions obtained in terms of the self-consistent dielectric function theory [3].

\section{Experimental constraints}

New measurements of the ${ }^{2} \mathrm{H}(d, p)^{3} \mathrm{H}$ and ${ }^{2} \mathrm{H}(d, n)^{3} \mathrm{He}$ reactions at energies ranging from 6 to $25 \mathrm{keV}$ were performed in $\mathrm{Zr}$ under UHV conditions. Impurities of the target surface, as well as the deuteron density have been continuously monitored. To justify experimentally observed 
cross section enhancement it was necessary to include an additional contribution resulting from a hypothetical single-particle $0^{+}$threshold resonance in ${ }^{4} \mathrm{He}$ compound nucleus [8]. The resonance component had to be taken into account as a coherent superposition with transition matrix elements for the dd reactions. It allowed for reduction of the experimentally obtained screening energy. However, its absolute value still overestimates the theoretical limit and can additionally be influenced by a number of crystal lattice defects of the metallic target.

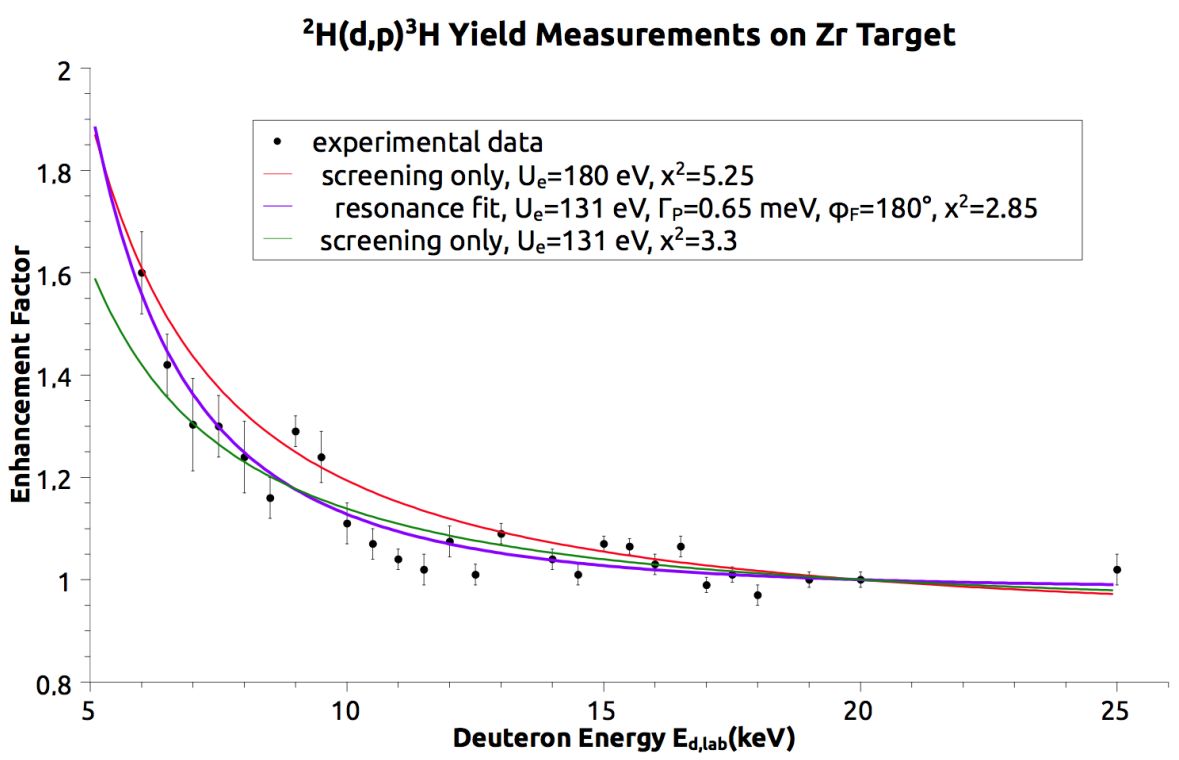

Figure 3: Enhancement factor for $\mathrm{d}+\mathrm{d}$ reactions on $\mathrm{Zr}$ target under UHV conditions. The experimental data pattern is modeled best taking into account both screening energy and resonance contribution. $U_{e}$ values are closer to the theoretical value, which is equal to $112 \mathrm{eV}$.

In the Figure 3 the experimental results for enhancement factor are displayed. Accurately determined screening enhancement factor vs. deuteron energy deviates significantly from the singleparametric curve fitted only with the screening energy. Moreover, target impurities, crystal lattice defects and effective electron mass can increase the measured screening energy, while target surface contamination leads to its reduction.

Additionally, a 22-hours measurement of the enhancement factor for deuteron energy of $15 \mathrm{keV}$ has been performed (Figure 4). A noticeable increase in measured enhancement corresponds presumably to crystal lattice defects of zirconium target, that were caused by beam irradiation for 4-5 hours. Estimated target surface impurity amounts to about 1 or 2 atomic monolayers. Serious contamination of the target surface triggers significant decrease in the enhancement factor.

\section{Conclusions}

The screening energies in zirconium obtained in the new measurements are significantly closer to the theoretical value of $112 \mathrm{eV}$. This can be caused by crystal lattice defects leading to higher effective electron mass. 


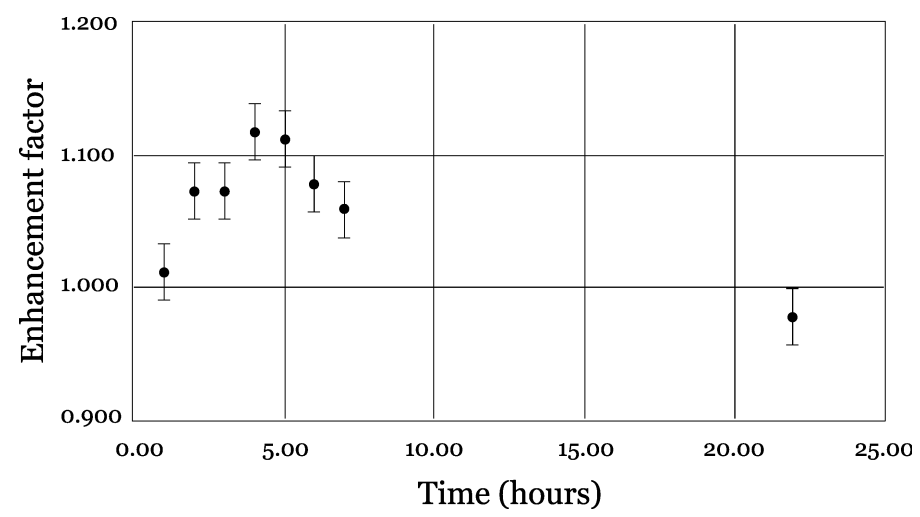

Figure 4: Long-time measurement of the enhancement factor for deuteron energy of $15 \mathrm{keV}$. After 4-5 hours of measurement the enhancement reaches its maximum due to crystal lattice defects. The corresponding target surface contamination amounts to 1-2 monolayers.

Furthermore, an improved energy dependence of the enhancement factor could be obtained under cleaner conditions, which deviates from screening-only fits. This can be explained by a destructive single-particle resonance interference contribution to the cross section.

To study all effects that influence the enhancement factor both the target contamination suppression and the beam intensity/stability should be further improved. Experimental data below $5 \mathrm{keV}$ are needed to determine the resonance contribution.

\section{References}

[1] K. Czerski et al., Europhys. Lett. 54, 449 (2001)

[2] K. Czerski et al., Europhys. Lett. 68, 363 (2004)

[3] A. Huke et al., Phys. Rev. C 78, 015803 (2008)

[4] J. Kasagi et al., J. Phys. Soc. Jpn. 71, 2281 (2002)

[5] C. Bonomo et al., Nucl. Phys. A 719, 37c (2003)

[6] F. Raiola et al., Eur. Phys. J. A 19, 283 (2004)

[7] K. Czerski et al., J. Phys. G 35, 014012 (2008)

[8] N. Targosz-Sleczka et al., PoS (NIC-X), 220 (2008)

[9] R.E. Brown and N. Jarmie, Phys. Rev. C 41, 1391 (1990)

[10] J.F. Ziegler et al., The Stopping and Ranges of Ions in Matter, Pergamon Press, NY 1985 